\title{
Zonulin, inflammation and iron status in patients with early stages of chronic kidney disease
}

\author{
Ewelina Lukaszyk $^{1} \cdot$ Mateusz Lukaszyk $^{2} \cdot$ Ewa Koc-Zorawska $^{1} \cdot$ Anna Bodzenta-Lukaszyk ${ }^{2} \cdot$ Jolanta Malyszko $^{1}$
}

Received: 12 August 2017 / Accepted: 6 November 2017 / Published online: 13 November 2017

(c) The Author(s) 2017. This article is an open access publication

\begin{abstract}
Background/aims Zonulin is the only known regulator of intestinal permeability. It is also considered as a potential inflammatory marker in several conditions such as diabetes and inflammatory bowel syndrome. The aim of the study was to investigate zonulin levels in patients with early stages of CKD and its possible correlation with inflammation, anemia and iron status parameters.

Methods Eighty-eight patients with early stages of CKD and 23 healthy volunteers were enrolled in the study. Zonulin, hepcidin-25, soluble transferrin receptor, interleukin-6 and high-sensitivity C-reactive protein were measured using commercially available assays.

Results Zonulin was significantly lower among patients with CKD in comparison with healthy volunteers. There were no statistically significant differences in zonulin concentration between patients with and without inflammation. Zonulin was significantly correlated with hepcidin only in patients with inflammation. Zonulin was neither related to iron nor related to ferritin.

Conclusions Zonulin cannot be considered as an inflammatory marker in CKD. It does not play a role in the disturbances of iron metabolism in CKD. Its physiological role remains to be elucidated.
\end{abstract}

Keywords Zonulin · Iron · Inflammation · Chronic kidney disease $\cdot$ Hepcidin

\section{Introduction}

Inflammation is one of the pathomechanisms responsible for progressive impairment in kidney function and is associated with common complications in chronic kidney disease (CKD) such as anemia. Inflammation also affects iron metabolism and is responsible for deranged iron distribution despite adequate iron stores in the body. Iron absorption takes place in the small intestine, where zonulin is the only known modulator of intracellular tight junction [1]. Zonulin has been identified as pre-haptoglobin 2 and regarded only as an inactive precursor for haptoglobin 2 (HP2). Zonulin is also considered as a potential inflammatory marker [2].

Jolanta Malyszko

jolmal@poczta.onet.pl

1 2nd Department of Nephrology and Hypertension with Dialysis Unit, Medical University of Bialystok, Marii Skłodowskiej-Curie 24a, 15-276 Białystok, Poland

2 Department of Allergy and Internal Medicine, Medical University of Bialystok, Białystok, Poland
Intestinal permeability and zonulin disturbances have been reported to be associated with diabetes type 1 [3], type 2 [4, 5], inflammatory bowel syndrome [6] and other conditions with low-grade inflammation [7].

In our previous study on small group of patients in second and third stage of CKD, we observed correlations of zonulin with interleukin-6 (IL-6) and hemojuvelin [8]. Therefore, we aimed to study zonulin levels in a larger population of patients with early stages of CKD and investigate its possible correlation with inflammation, anemia and iron status.

\section{Patients and methods}

Eighty-eight patients with CKD (defined as: an estimated glomerular filtration rate $(\mathrm{eGFR}) \leq 60 / \mathrm{ml} / \mathrm{min} / 1.75 \mathrm{~m}^{2}$ or presence of hematuria or proteinuria for $\geq 3$ month) were enrolled into the study and divided into two groups-with and without subclinical inflammation according to hsCRP measurements. Zonulin concentration was also measured in the healthy volunteers $(n=23)$. Patients with clinically 
evident inflammation, acute thrombosis, active malignancy, acute cardiovascular complications (including uncontrolled hypertension, acute coronary syndrome and acute heart failure), anemia and/or iron deficiency treatment, blood transfusions within 3 months prior the study and immunosuppressive therapy were excluded from the study. Every subject gave informed consent, and the study protocol was approved by the Medical University Ethics Committee.

Medical history (including demographic characteristics as well as current pharmacotherapy) and blood samples were collected in all subjects at the time of enrollment in the morning after an overnight fast. Hematological measurements were taken using fresh venous blood with EDTA and clotted blood. The plasma and serum were centrifuged and frozen at $-70{ }^{\circ} \mathrm{C}$ until further laboratory analysis.

Serum hemoglobin, creatinine, iron, total iron binding capacity (TIBC) and ferritin levels were obtained using standard laboratory methods (automated system) in certified local central laboratory. Transferrin saturation (TSAT) was calculated as the ratio of serum iron and TIBC and expressed as a percentage. Glomerular filtration rate was estimated with the use of Chronic Kidney Disease Epidemiology Collaboration formula (CKD-EPI).

Zonulin was assessed using kit from Immundiagnostik, Germany. Hepcidin-25 was measured using an assay from Bachem, UK. Soluble transferrin receptor-sTfR and interleukin-6 were studied by using kits from R\&D Systems, UK. High-sensitivity C-reactive protein-hsCRP was measured with the use of CRP ELISA Kit LDN Labor Diagnostika Nord GmbH\&Co KG, Germany.

Data with normal distribution were reported as mean \pm standard deviation, and non-normally distributed as median and interquartile range. Variables with skewed distribution were $\log (\ln )$-transformed before further statistical analysis. Analyses of the correlation of each parameter were performed using Pearson or Spearman correlation coefficients. The multiple regression analysis was used to determine independent factors affecting the dependent variables. Factors showing linear correlation with zonulin or hepcidin $(p<0.05)$ were included in the analysis. All statistical analyses were performed using Statistica 12.5 (StatSoft) computer software.

\section{Results}

Patients' laboratory characteristic is presented in Table 1. Zonulin was significantly lower in patients with CKD than in healthy volunteers $(38.2 \pm 16$ vs $48.5 \pm 6.4 \mathrm{ng} / \mathrm{mL}$; $p=0.003$ ). There is a trend toward decrease in zonulin levels with the severity of CKD (Fig. 1). There were no statistically significant differences in zonulin concentration between patients with and without inflammation as well as anemic and non-anemic patients with CKD. Patients with inflammation had significantly lower hemoglobin, iron and TSAT values. Hepcidin concentration was higher in patients with inflammation.

Zonulin was significantly correlated with hepcidin $(r=-0.37, p<0.05)$ only in patients with inflammation defined as elevated hsCRP $>10 \mathrm{mg} / \mathrm{dL}$ (Fig. 2). Hepcidin was correlated with TSAT $(r=0.49, p<0.05)$, ferritin $(r=0.73, p<0.05)$ and $\operatorname{sTfR}(r=-0.41, p<0.05$-Fig. 3$)$ in both groups as well as in independent analysis. Zonulin was not related to iron or ferritin.

\section{Discussion}

In this study, we found that zonulin correlated significantly with hepcidin in patients with early stages of CKD and

Table 1 Characteristic of patients with chronic kidney disease and healthy control

\begin{tabular}{|c|c|c|c|c|c|}
\hline & $\begin{array}{l}\text { CKD patients } \\
\text { with inflammation } \\
\text { hsCRP }>10 \mathrm{mg} / \mathrm{dL} \\
n=35\end{array}$ & $\begin{array}{l}\text { CKD patients with } \\
\text { no inflammation } \\
\text { hsCRP }<10 \mathrm{mg} / \mathrm{dL} \\
n=53\end{array}$ & Control $n=23$ & $\begin{array}{l}p \text { value (inflamma- } \\
\text { tion vs no inflamma- } \\
\text { tion) }\end{array}$ & $\begin{array}{l}p \text { value (CKD with no } \\
\text { inflammation vs control) }\end{array}$ \\
\hline Age, years & $73.9 \pm 10.9$ & $68 \pm 11.3$ & $52 \pm 9.0$ & 0.02 & $<0.001$ \\
\hline Hemoglobin, g/dL & $12.2 \pm 2.1$ & $13.7 \pm 2.0$ & $14 \pm 1.0$ & $<0.001$ & 0.5 \\
\hline Iron, $\mu \mathrm{g} / \mathrm{dL}$ & $54.2 \pm 28.0$ & $86.9 \pm 35.2$ & $95.7 \pm 25$ & $<0.001$ & 0.28 \\
\hline TSAT, $\%$ & $23.2 \pm 12.1$ & $30.3 \pm 13.4$ & $29.1 \pm 7$ & 0.01 & 0.69 \\
\hline Ferritin, mg/dL & $171.0(75.6-339.8)$ & $123.1(90.3-190.9)$ & 104 61-159) & 0.2 & 0.1 \\
\hline $\mathrm{eGFR}, \mathrm{mL} / \mathrm{min} / 1.73 \mathrm{~m}^{2}$ & $60.8 \pm 20.1$ & $66.2 \pm 18.1$ & $98.1 \pm 16$ & 0.2 & $<0.001$ \\
\hline IL-6, pg/mL & $4.1(1.04-12.2)$ & $0.8(0.22-3.4)$ & $0.5(0.1-0.9)$ & $<0.001$ & 0.2 \\
\hline Hepcidin-25, ng/mL & $41.7(25.1-87.9)$ & $35.4(18.2-47.4)$ & $24.5(19.1-33)$ & 0.04 & 0.36 \\
\hline Zonulin, ng/mL & $36.6 \pm 20.6$ & $37.3 \pm 12.1$ & $48.5 \pm 6.4$ & 0.84 & $<0.001$ \\
\hline $\mathrm{sTfR}, \mathrm{nmol} / \mathrm{L}$ & $19.0(16.4-26.1)$ & $17.1(14.4-23.3)$ & $10(8-11)$ & 0.13 & 0.02 \\
\hline hsCRP, mg/dL & $25.2(18.9-40.9)$ & $3.9(1.6-5.9)$ & $0.9(0.3-1.4)$ & $<0.000$ & 0.01 \\
\hline
\end{tabular}


Fig. 1 Zonulin concentrations in CKD patients. One-way ANOVA, $\mathrm{F}(3,84)=0.62$ $p=0.85$ vs control group $F(4,1050)=3.26, p=0.014$

Fig. 2 Correlation between zonulin and hepcidin in patients with CKD and inflammation, $r=-0.37$

Fig. 3 Correlation between hepcidin-25 and sTfR in patients with CKD, $r=-0.41$
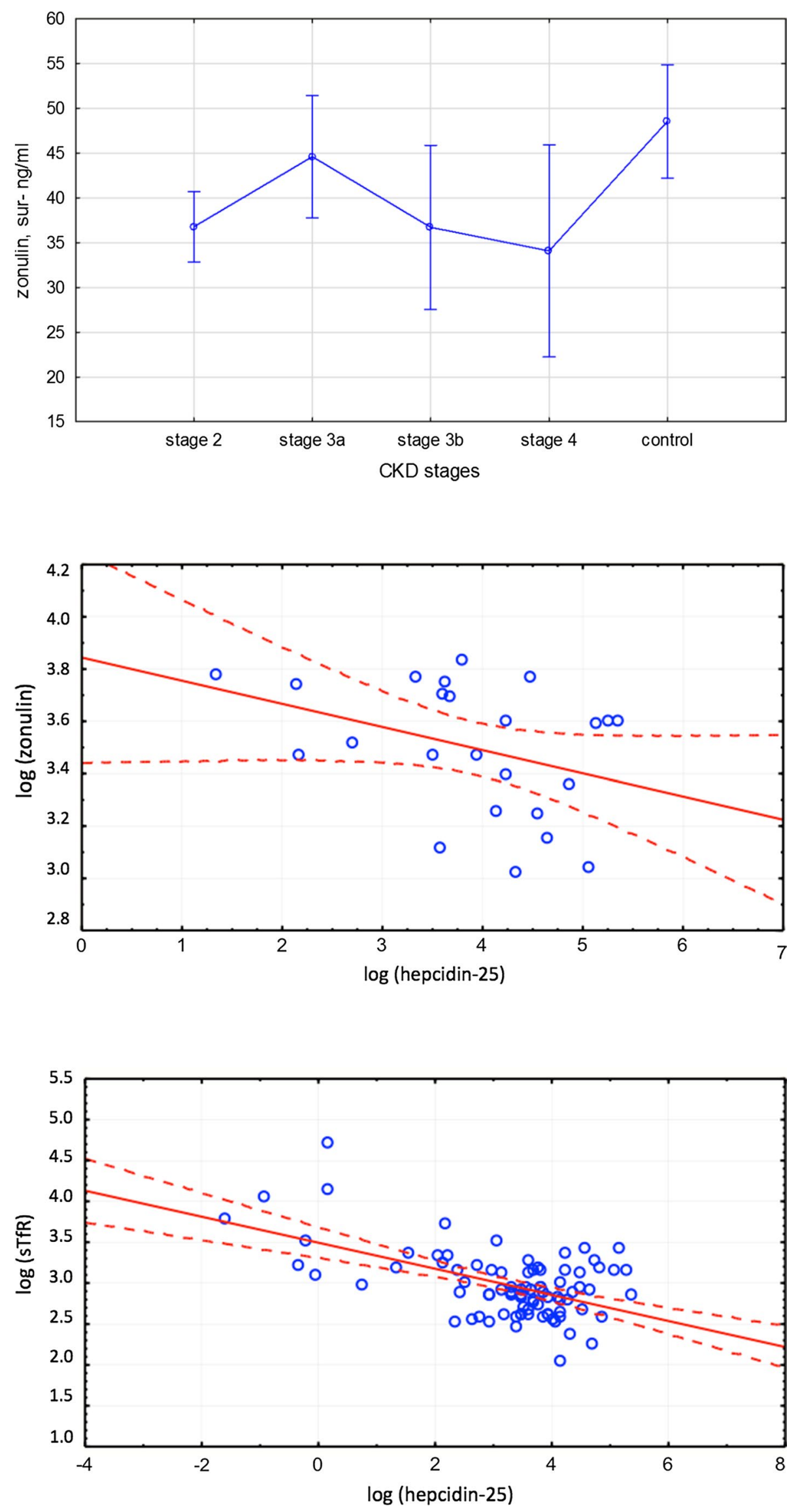
inflammation defined as elevated hsCRP $>10 \mathrm{mg} / \mathrm{dL}$. There were no differences in zonulin concentration between anemic and non-anemic patients with CKD as well as patients with and without inflammation. We did not find any significant correlations between zonulin and iron status parameters.

Zonulin is known as a mediator regulating intestinal permeability throughout disassembling intracellular tight junctions [1]. In recent years, tight junctions and zonulin begin to emerge in a spotlight of many areas of medicine including autoimmune diseases, malignancy and disorders of central nervous system [9]. In addition, zonulin overexpression in the immune-mediated diseases such as diabetes mellitus type 1 and celiac disease $[10,11]$ suggests that zonulin is an inflammatory marker, like its precursor, haptoglobin [4], a liver acute-phase response protein. Moreover, the expression of haptoglobin in hepatocytes is increased by number of proinflammatory cytokines, including interleukin-1, interleukin-6 and tumor necrosis factor alpha [12]. Circulating zonulin is also considered to be a potential marker of intestinal permeability in dermatitis herpetiformis [13].

Malyszko et al. [14] assessed serum zonulin levels in kidney transplant recipients. Serum zonulin was significantly lower than in healthy controls. However, zonulin was not related to iron parameters studied. Zonulin concentration was also measured in heart transplant recipients [15] and was significantly lower than in the healthy volunteers. In both studies, zonulin was not related to any of the iron status parameters that is consistent with the results of our study. Authors suggest that low zonulin concentration might occur due to impaired defensive mechanisms caused by immunosuppressive therapy $[14,15]$.

In our study, a moderate increase in inflammatory markers, typical for CKD, was found. As reported previously by Kotanko et al. [16], degree of systemic inflammation in CKD corresponded to the level of endotoxemia. In sepsis, elevated zonulin levels have been described, probably due to the link between systemic inflammatory response syndrome and increased intestinal permeability [17]. Recently, it has also been reported that higher concentrations of zonulin were associated with the insulin resistance and severity of menstrual disorders in patients with polycystic ovary syndrome (PCO) [18]. Interestingly, in murine models, zonulin has been associated with development of acute lung injury by enhancing albumin leak, complement activation and increased cytokines and neutrophils production [19].

In the recent study by Ficek et al. [20] in hemodialyzed patients, a weak correlation between levels of IL-6 and D-lactates and lipolysaccharide-LPS and the greatest severity of inflammation were found in the highest tertile of zonulin, LPS and D-lactates concentrations, while in multiple regression models, serum hsCRP and plasma IL-6 variability were explained by LPS only. They also stressed that the weak correlations between zonulin and inflammatory parameters suggesting that the other wellknown mechanisms of systemic inflammation in HD played a predominant role. Recently, zonulin was assessed in 225 patients carrying automatic implantable cardioverters/defibrillators (AICD) for primary or secondary prevention [21]. The inverse associations between zonulin and creatinine as well as markers of cardiovascular risk (high calprotectin and kynurenine, low homoarginine) were reported. In addition, the authors found that in the subgroup with type 2 diabetes, zonulin increased significantly together with high-sensitivity CRP. Higher circulating zonulin concentrations have been found in subjects with impaired glucose tolerance regardless of body mass [7]. Zonulin was also significantly elevated in patients with type 2 diabetes [5] and associated with inflammatory markers and poor glycemic control [4]. In our studied group, 23 patients were diabetic; however, we did not find statistically significant difference in zonulin levels between diabetic vs non-diabetic subjects $(42.9 \mathrm{ng} / \mathrm{mL}$ in diabetes vs $36.5 \mathrm{ng} / \mathrm{mL}$ in non-diabetes patients with CKD, $p=0.09$ ).

There are several limitations of the study. The sample is not large to make definite conclusions, and there is a need for a larger sample and other groups of patients to follow prospectively to conclude whether zonulin as a biomarker will help to distinguish inflammation in CKD. It also should be stressed that our study might be underpowered to show an association and therefore further research are needed to prove or disprove the causality of zonulin and inflammation in early CKD.

Very recently, Vojdani et al. [22] stressed the problem of zonulin variability. Zonulin is a protein the size of 47,000 Da, released from the lamina propria and presented to the submucosal gut immune system [23]. Then, zonulin-specific antibodies are synthesized as an immune response to many environmental factors [23]. According to Vojdani et al. [22], this immune response against zonulin and other large molecules may be an explanation for zonulin fluctuation. As majority of laboratories analyze serum zonulin levels from a single blood draw, variability of serum zonulin levels during the time course of a single day or from day to day should be taken into account. Thus, different studies yielding not comparable results should bear in mind the zonulin variability.

In conclusion, zonulin appears not to be an inflammatory marker in CKD. It seems it also does not play a role in the disturbances of iron metabolism in CKD. Its physiological role remains to be elucidated.

Acknowledgements The study was funded by the University Grant for Young Investigators (EL). This study was conducted with the use of equipment purchased by Medical University of Bialystok as part of the RPOWP 2007-2013 funding, Priority I, Axis 1.1, contract No. UDA-RPPD.01.01.00-20-001/15-00 dated 26.06.2015. 


\section{Compliance with ethical standards}

Conflict of interest All authors declare that they have no conflict of interest.

Informed consent Informed consent was obtained from all individual participants included in the study.

Open Access This article is distributed under the terms of the Creative Commons Attribution 4.0 International License (http://creativecommons.org/licenses/by/4.0/), which permits unrestricted use, distribution, and reproduction in any medium, provided you give appropriate credit to the original author(s) and the source, provide a link to the Creative Commons license, and indicate if changes were made.

\section{References}

1. Fasano A (2009) Regulation of intercellular tight junctions by zonula occludens toxin and its eukaryotic analogue zonulin. Ann N Y Acad Sci 915:214e22

2. Tripathi A, Lammers KM, Goldblum S, Shea-Donohue T, NetzelArnett S, Buzza MS, Antalis TM, Vogel SN, Zhao A, Yang C, Arrietta MC, Meddings JB, Fasano A (2009) Identification of human zonulin, a physiological modulator of tight junction, as prehaproglobin-2. Proc Natl Acad Sci USA 106:16799

3. Sapone A, de Magistris L, Pietzak M, Clemente MG, Tripathi A, Cucca F, Lampis R, Kryszak D, Cartenì M, Generoso M, Iafusco D, Prisco F, Laghi F, Riegler G, Carratu R, Counts D, Fasano A (2006) Zonulin upregulation is associated with increased gut permeability in subjects with type 1 diabetes and their relatives. Diabetes 55:1443-1449

4. Jayashree B, Bibin YS, Prabhu D, Shanthirani CS, Gokulakrishnan K, Lakshmi BS, Mohan V, Balasubramanyam M (2014) Increased circulatory levels of lipopolysaccharide (LPS) and zonulin signify novel biomarkers of proinflammation in patients with type 2 diabetes. Mol Cell Biochem 388:203-210

5. Zhang D, Zhang L, Zheng Y, Yue F, Russell RD, Zeng Y (2014) Circulating zonulin levels in newly diagnosed Chinese type 2 diabetes patients. Diabetes Res Clin Pract 106:312-318

6. Vanuytsel T, Vermeire S, Cleynen I (2013) The role of Haptoglobin and its related protein, Zonulin, in inflammatory bowel disease. Tissue Barriers 1:e27321

7. Moreno-Navarrete JM, Sabater M, Ortega F, Ricart W, FernándezReal JM (2012) Circulating zonulin, a marker of intestinal permeability, is increased in association with obesity-associated insulin resistance. PLoS ONE 7:e37160

8. Łukaszyk E, Łukaszyk M, Koc-Żórawska E, Tobolczyk J, Bodzenta-Łukaszyk A, Małyszko J (2015) Iron status and inflammation in early stages of chronic kidney disease. Kidney Blood Press Res 40:366-373

9. Fasano A (2011) Zonulin and its regulation of intestinal barrier function: the biological door to inflammation, autoimmunity, and cancer. Physiol Rev 91:151-175
10. Arrieta MC, Bistritz L, Meddings JB (2006) Alterations in intestinal permeability. Gut 55:1512-1520

11. Fasano A, Catassi C (2001) Current approaches to diagnosis and treatment of celiac disease: an evolving spectrum. Gastroenterology 120:636-651

12. Baumann H, Morella KK, Wong GH (1993) TNF-alpha, IL-1 beta, and hepatocyte growth factor cooperate in stimulating specific acute phase plasma protein genes in rat hepatoma cells. J Immunol 151:4248-4257

13. Smecuol E, Sugai E, Niveloni S, Vázquez H, Pedreira S, Mazure R, Moreno ML, Label M, Mauriño E, Fasano A, Meddings J, Bai JC (2005) Permeability, zonulin production, and enteropathy in dermatitis herpetiformis. Clin Gastroenterol Hepatol 3:335-341

14. Malyszko J, Koc-Zorawska E, Levin-Iaina N, Malyszko J (2014) Zonulin, iron status, and anemia in kidney transplant recipients: are they related? Transplant Proc 46:2644-2646

15. Przybyłowski P, Nowak E, Janik L, Wasilewski G, Kozlowska S, Małyszko J (2014) Zonulin and iron metabolism in heart transplant recipients. Transplant Proc 46:2856-2859

16. Kotanko P, Carter M, Levin NW (2006) Intestinal bacterial microflora-a potential source of chronic inflammation in patients with chronic kidney disease. Nephrol Dial Transplant 21:2057-2060

17. Klaus DA, Motal MC, Burger-Klepp U, Marschalek C, Schmidt EM, Lebherz-Eichinger D, Krenn CG, Roth GA (2013) Increased plasma zonulin in patients with sepsis. Biochem Med (Zagreb) 23:107-111

18. Zhang D, Zhang L, Yue F, Zheng Y, Russell R (2015) Serum zonulin is elevated in women with polycystic ovary syndrome and correlates with insulin resistance and severity of anovulation. Eur J Endocrinol 72:29-36

19. Rittirsch D, Flierl MA, Nadeau BA, Day DE, Huber-Lang MS, Grailer JJ, Zetoune FS, Andjelkovic AV, Fasano A, Ward PA (2013) Zonulin as prehaptoglobin2 regulates lung permeability and activates the complement system. Am J Physiol Lung Cell Mol Physiol 304:L863-872

20. Ficek J, Wyskida K, Ficek R, Wajda J, Klein D, Witkowicz J, Rotkegel S, Spiechowicz-Zatoń U, Kocemba-Dyczek J, Ciepał J, Więcek A, Olszanecka-Glinianowicz M, Chudek J (2017) Relationship between plasma levels of zonulin, bacterial lipopolysaccharides, D-lactate and markers of inflammation in haemodialysis patients. Int Urol Nephrol 49:717-725

21. Dschietzig TB, Boschann F, Ruppert J, Armbruster FP, Meinitzer A, Bankovic D, Mitrovic V, Melzer C (2016) Plasma zonulin and its association with kidney function, severity of heart failure, and metabolic inflammation. Clin Lab 62:2443-2447

22. Vojdani A, Vojdani E, Kharrazian D (2017) Fluctuation of zonulin levels in blood vs stability of antibodies. World J Gastroenterol 23:5669-5679

23. Fasano A, Not T, Wang W, Uzzau S, Berti I, Tommasini A, Goldblum SE (2000) Zonulin, a newly discovered modulator of intestinal permeability, and its expression in coeliac disease. Lancet 355:1518-1519 